\title{
Optimisation of Image Fusion using Feature Matching Based on SIFT and RANSAC
}

\author{
Gurpreet Kaur* and Prateek Agrawal
}

\author{
School of Computer Science Engineering, Lovely Professional University, Phagwara - 144411, Punjab, India;
} gurpreetkaur2091@gmail.com, prateek061186@gmail.com

\begin{abstract}
Background/Objectives: Image fusion is the technique which merges the input images to obtain the focused single image. A new method is proposed in this paper to optimise image fusion using feature matching based Scale Invariant Feature Transform (SIFT) and Random Sample Consensus (RANSAC). Methods/Analysis: In our proposed method, two inputimages are fused using Stationary Wavelet Transform to get a single focused image. Then, feature matching technique called SIFT is applied to match the corresponding features between two images. Further, RANSAC is applied to further optimise the result of SIFT and get a final fused image. Findings: Quantitative and visual results show that a highly focused and better fused image is obtained after feature matching with SIFT and further refinement with RANSAC. The proposed method is robust and independent of scale, light intensity, orientation of camera etc. Applications: The methodology for image fusion may be applied to stereo-images. Feature matching based on SIFT and RANSAC may be used to reconstruct a 3D view from stereo-images.
\end{abstract}

Keywords: Image Fusion, RANSAC, SIFT, Stationary Wavelet Transform

\section{Introduction}

Multi-focus fusion algorithm combines two input images to obtain a focused single image. The real focus of fusion of images is to get a fused image having higher quality and information in comparison to the input images. A sharp image containing all focused objects cannot be obtained because optical lenses have a limited depth of field. So, image fusion is being utilised in which a group of images can be acquired using differently adjusted focus settings. These images are then combined to get an image with extended field depth. It is utilized in many applications like remote sensing $\underline{1}$, image processing, computer vision, medical imaging 2 , object recognition etc. The image fusion technique occurs at various levels as decision, feature and pixel levels. The pixel based level is the lowest base level of image fusion which handles pixels obtained by imaging with sensor output. This based level enhances the visual based enhancement. At pixel level, image fusion process takes intensity of source images into consideration. The benefits of pixel level fusion are detection of undesirable noise, easy implementation and low complexity. However, these methods have disadvantages of mis-registration and blurred artifacts. To handle the problems of noise sensitivity and mis-registration, region based methods can be utilised. In feature level based fusion technique, extraction of features is being done from the input images, image based segmentation is being done in continuous regions followed by fusion. Image features like shape, contrast, size are integrated in feature based level. Decision level based fusion technique handles the descriptors of image. In comparison to decision level and region based image fusion, pixel level based image fusion

${ }^{*}$ Author for correspondence 
gives relatively more complete and detailed information. Image fusion technique is categorised into spatial and frequency domains. Spatial based domain deals with image pixels directly and merges the required information to achieve the desired objective. Techniques such as Brovey method ${ }^{3}$, Principal Component Analysis ${ }^{4}$, Intensity Hue Saturation ${ }^{5}$, high pass filtering are the methods based on spatial domain. In frequency based domain, input image is initially transferred to the frequency based domain and the resulting technique of image fusion is acquired by merging the frequency based coefficients. Pyramid based methods like ratio of low pass pyramid ${ }^{6}$, laplacian pyramid $^{7}$ and wavelet based techniques such as Discrete Wavelet Transform ${ }^{8}$, Stationary Wavelet Transform ${ }^{9}$, Haar wavelet ${ }^{10}$, Daubechies wavelets ${ }^{11}$ are categorised under the frequency domain based methods. Basically image regis- tration $\frac{12}{2}$ is followed by image fusion. Image registration is the technique of transforming various images into the system having similar coordinates. Fusion techniques are basically problem dependent. Various focus measures $\underline{\underline{13}}$ for analyzing the performance of multi-focus image fusion include energy of gradient, variance, sum-modified-laplacian, energy of laplacian of image.

\section{Algorithms}

\subsection{Stationary Wavelet Transform}

Stationary Wavelet Transform (SWT) is a shift variant and non-orthogonal transform. Since, sub-sampling is not performed in stationary wavelet transform, resolution of signal do not decrease as approximation and detail

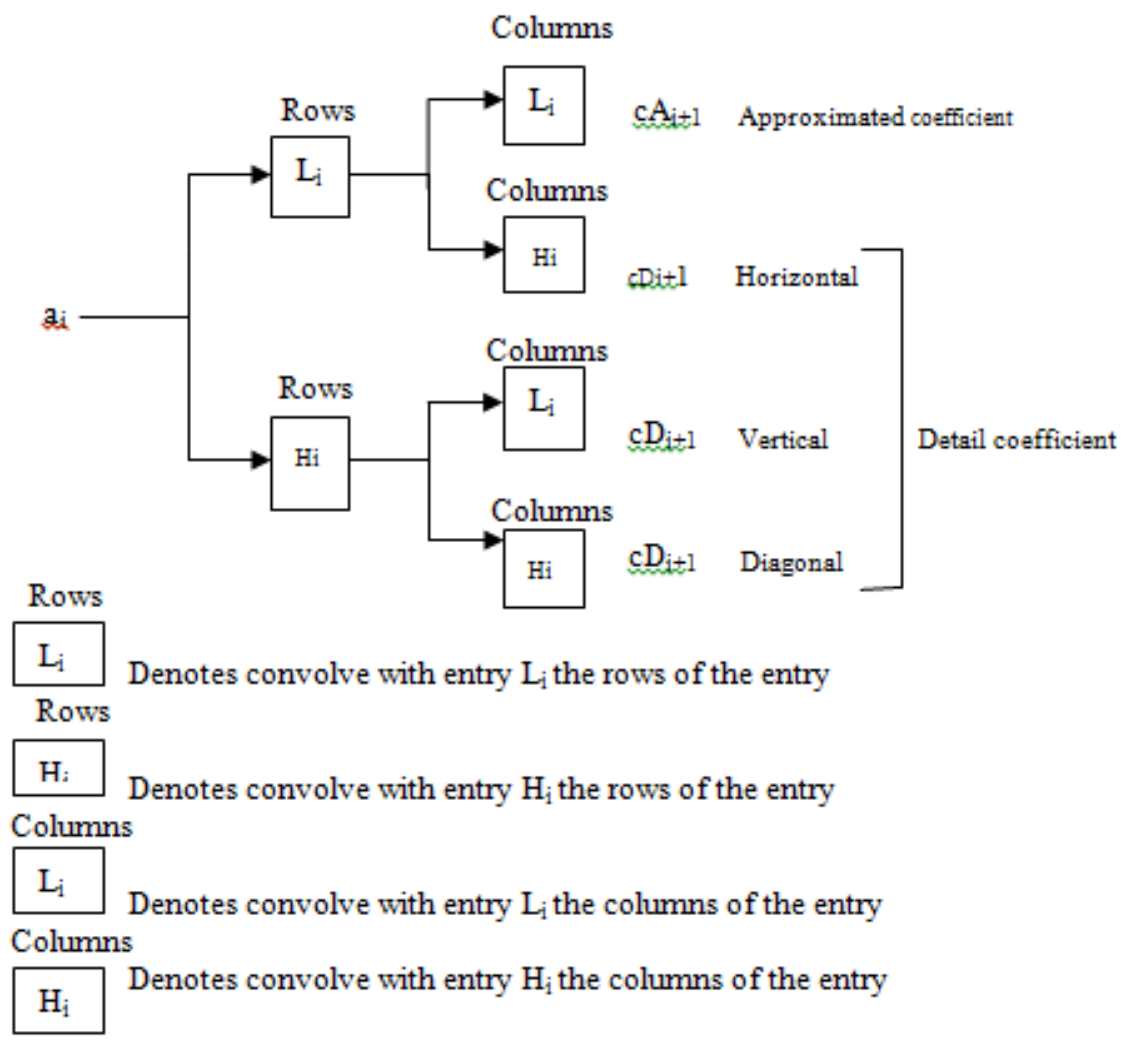

Figure 1. Decomposition Procedure of Stationary Wavelet Transform. 
coefficients length remain same as that of the original signal. Initially, the source images are decomposed in stationary wavelet transform wavelet basis at level $\mathrm{N}$ to get approximation and detail coefficients? . Decomposition process in SWT is shown in Figure 1. A threshold and shrinkage function is chosen for the coefficients at each level. Focused regions of each source image are obtained in this step. Finally, new coefficients are chosen for the reconstruction of source image using inverse stationary wavelet transform, which transforms coefficient presentation into an image.

\subsection{Scale Invariant Feature Transform}

Scale Invariant Feature Transform (SIFT) is the technique of detection and matching of feature point formulated in 1999 by Lowe $e^{14}$. SIFT features are not variant for scaling, rotation, translation, affine and change in illumination of image $\frac{15}{}$. Its applications are in fields of object recognition, image mosaic, localization of mobile robots etc. SIFT feature points extraction consists of following four steps:

1. Detection of extreme of Scale-space: The extreme points of scale space are selected as candidate matching feature image points in SIFT algorithm. Image $J(i, j)$ scale space is defined

$\mathrm{S}(\mathrm{m}, \mathrm{n}, \mathrm{\sigma})=\mathrm{J}(\mathrm{m}, \mathrm{n})^{\star} \mathrm{G}(\mathrm{m}, \mathrm{n})$

where ${ }^{*}$ is the two dimensional convolution, $G(m, n)$ is a Gaussian function, $\sigma$ is the standard deviation of normal Gaussian distribution. The extreme is detected in image convolution and difference of Gaussian (DoG).

2. Localization of key points: Taylor expansion is constructed as DoG function in scale space. . In this process, key-point candidates were produced in large number, out of which some were unstable. Then the detailed fit is performed for the adjacent data to compute scale accuracy, location and the ratio of the principal curvatures. The computed data is used to reject points with low contrast, high noise sensitivity and poor localization along an edge.

Lowe mentioned that the location of the extrema to the accuracy of sub-pixels is done by fitting a three dimensional quadratic function to the laplacian based scale space. Taylor expansion based on the function of scale space $\mathrm{D}(\mathrm{p}, \mathrm{q}, \sigma)$ which is shifted for aligning the origin to the point of interest is used in this approach.

3. Orientation assignment: Each feature point is assigned a main direction, with magnitude $\mathrm{M}(\mathrm{m}, \mathrm{n})$ and gradient direction $\Theta(\mathrm{m}, \mathrm{n})$.

$$
\begin{aligned}
& M(i, j)=\left[(S(m, n+1)-S(m, n-1))^{2}+(S(m+1, n)-S(m-\right. \\
& \left.1, n))^{2}\right]^{2}
\end{aligned}
$$

$$
\Theta(m, n)=\arctan \frac{S(m, n+1)-S(m, n-1)}{S(m+1, n)-S(m-1, n)}
$$

4. Descriptor of keypoint: The area around the critical point of image is divided into blocks, histogram is calculated for every block and the vector with 128 dimensions is generated.

\subsection{Random Sample Consensus}

The Random Sample Consensus (RANSAC) is the technique in which parameters are estimated to deal with a large amount of outliers in source data ${ }^{16}$. It is based on resampling in which solutions are generated for estimation of model parameters by using minimum number of observations. RANSAC makes use of the minimum possible set and continues to enlargen the set with consistency based data points. The basic steps of algorithm:

1. The least possible feature points are selected randomly for determining parameters of the model.

2. The model based parameters are evaluated. 
3. The number of points which fit with a predefined tolerance $€$ are determined from a set of points.

4. The model parameters are re-estimated if fraction of total inliers over total points in set exceeds the predefined value of threshold $\mathrm{T}$ and then terminated.

5. Otherwise, steps 1 to 4 are repeated for maximum $\mathrm{N}$ number of times.

\section{Proposed Algorithm}

The proposed method optimises image fusion process using feature matching based SIFT and further refinement with RANSAC. The images are fused using Stationary Wavelet Transform (SWT), SIFT is applied to extract features and then RANSAC is used to get more refined results. The proposed algorithm is robust and independent of scale, translation, rotation, change in light intensity and orientation of camera.
The technique is shown in Figure 2 and step details are mentioned below:

Step1:. Two partially blurred images are taken as input.

Step2: Image registration is applied on both input images.

Step3: Stationary Wavelet Transform is applied on input images.

Step4: Image fusion is done using stationary values of image and a fused image is obtained.

Step5: SIFT is applied to extract features.

Step6: RANSAC is applied to optimse the image obtained in step 5 .

Step7: Finally the resultant fused image is obtained based on feature matching performed in steps 6 and 7 .

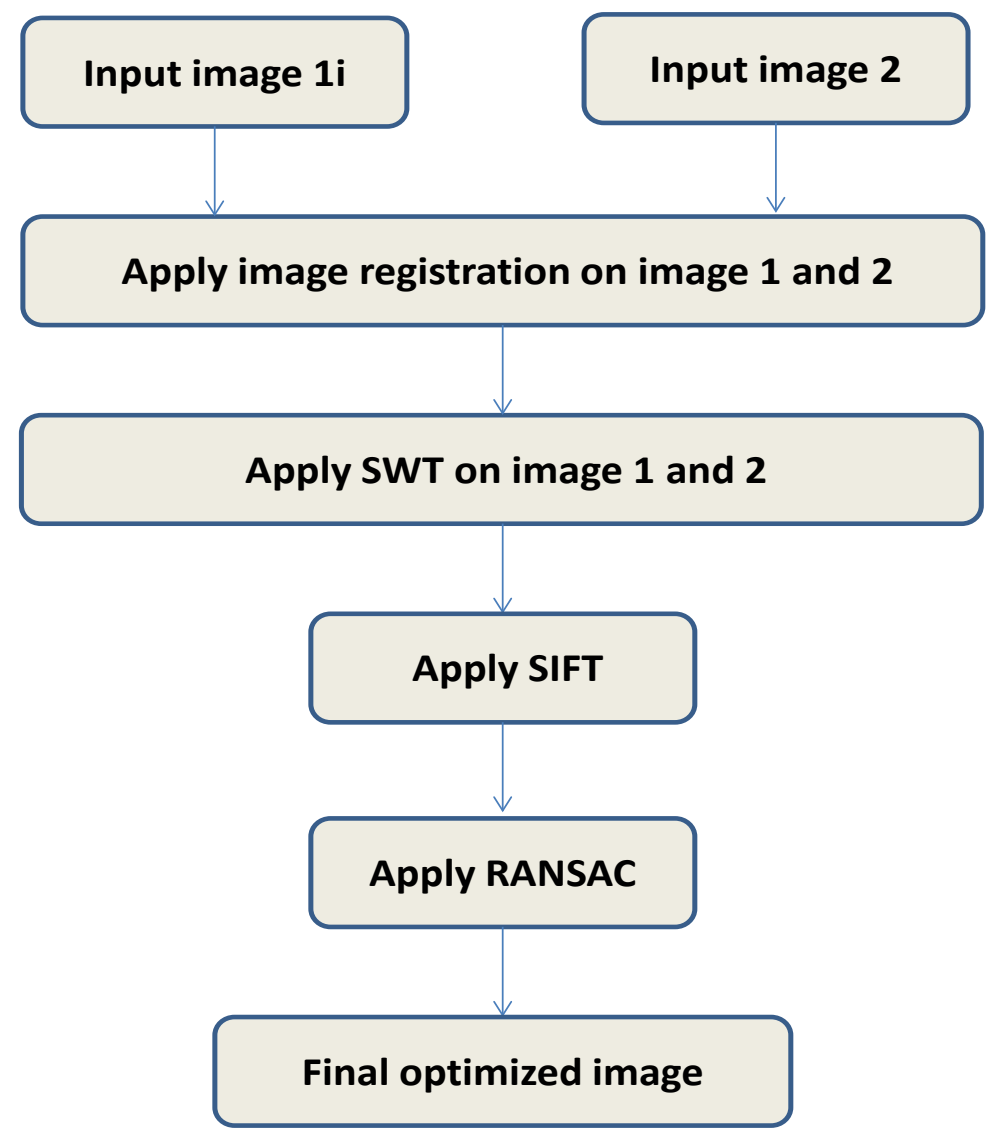

Figure 2. Proposed methodology. 


\section{Experimental Results}

The MATLAB 7.10.0 (R2010a) and image processing toolbox is used for implementation. Experimental results of existing and proposed techniques are shown below:

In Table 1, input image 1 and image 2 are left and right images taken from a stereo camera, SWT represents image formed by fusion of left and right input images using Stationary Wavelet Transform, SIFT represents the resultant image formed by feature matching using Scale Invariant Feature Transform, RANSAC represents optimised image formed by feature matching using Random Sample Consensus algorithm. It can be observed from the results that RANSAC optimises the feature matching done using SIFT by optimising the value of threshold.

Table 1. Experimental results

\begin{tabular}{|c|c|c|c|c|c|c|}
\hline Image name & Input image 1 & Input image 2 & SWT & SIFT & RANSAC & $\begin{array}{c}\text { Optimised } \\
\text { Image }\end{array}$ \\
\hline Coke & & & & & \\
\hline Shake & & & & & & \\
\hline
\end{tabular}


Table 1 Continued

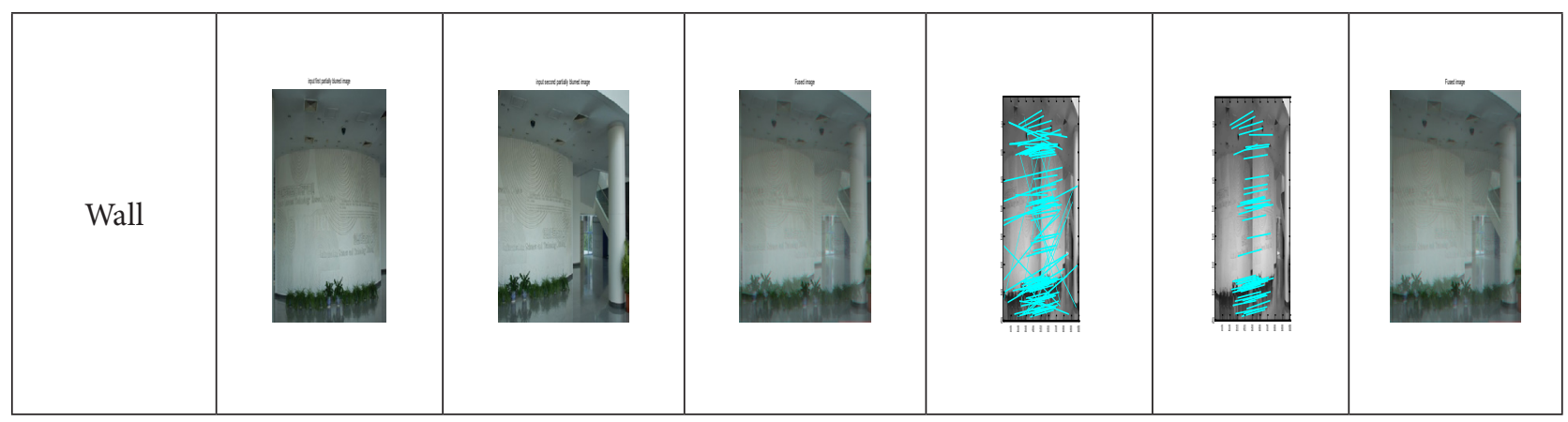

\section{Quantitative Results}

Performance evaluation is done on the basis of measures of spatial frequency, mean structural similarity index (MSSIM), peak signal to noise ratio (PSNR), mean square error (MSE), execution time, number of matches by SIFT and number of matches by RANSAC. It can be observed from the table 2 that number of matches by RANSAC is less than that of the number of matches by SIFT. So, RANSAC optimizes the feature matching of image by optimizing the value of threshold.

\section{Conclusion}

Image fusion is being considered as a wide area of research topic. It has various applications of remote sensing, imaging in medical field, computer vision etc. Images are fused using Stationary Wavelet Transform and feature matching is used for optimization. SIFT is used to extract features from image. RANSAC is used to further optimise and refine the results of SIFT. Feature matching is done based on the extracted features and the optimized resultant image is obtained. Feature matching based fusion

Table 2. Performance evaluation

\begin{tabular}{|c|c|c|c|c|c|c|c|}
\hline Image name & $\begin{array}{c}\text { Spatial } \\
\text { frequency }\end{array}$ & MSSIM & PSNR & MSE & $\begin{array}{c}\text { Execution } \\
\text { time }\end{array}$ & $\begin{array}{c}\text { No of } \\
\text { matches by } \\
\text { SIFT }\end{array}$ & $\begin{array}{c}\text { No of matches } \\
\text { by RANSAC }\end{array}$ \\
\hline Coke & 5.6325 & 0.8644 & 51.002 & 183 & 14.7667 & 149 & 17 \\
\hline Shake & 5.7228 & 0.8728 & 51.4452 & 174 & 8.0850 & 148 & 8 \\
\hline Tree & 6.1489 & 0.7048 & 38.4940 & 773 & 3.1443 & 160 & 93 \\
\hline Wall & 6.2024 & 0.6665 & 41.9022 & 522 & 1.9542 & 105 & 49 \\
\hline
\end{tabular}


makes the method robust and independent of change in illumination, orientation of camera etc.

\section{Future Work}

The methodology for image fusion may be applied to stereo-images. Feature matching based on SIFT and RANSAC may be used to reconstruct a $3 \mathrm{D}$ view from stereo-images.

\section{References}

1. Ghassemian H. A review of remote sensing image fusion methods. Information Fusion. 2016; 32: 75-89.

2. James A, Dasarathy B. Medical image fusion: A survey of the state of the art. Information Fusion.2014; 19: 4-19.

3. Selvarani P, Vaithyanat V. Coarse to Fine Level Set Segmentation of SAR Imagery Based on The Brovey Transform Fusion of Optical Imagery. Research Journal of Applied Sciences. 2012; 7(7): 334-9.

4. Wan T, Zhu C,Qin Z. Multifocus image fusion based on robust principal component analysis. Pattern Recognition Letters. 2013; 34(9): 1001-8.

5. Myungin Choi. A new intensity-hue-saturation fusion approach to image fusion with a tradeoff parameter. IEEE Transactions on Geoscience Remote Sensing. 2006; 44(6):1672-82.

6. Toet A. Image fusion by a ratio of low-pass pyramid. Pattern Recognition Letters. 1989; 9(4); 245-53.

7. Burt P, Adelson E. The Laplacian Pyramid as a Compact
Image Code. IEEE Transactions on Communications. 1983; 31(4):532-40.

8. Vijayarajan R, Muttan S. Discrete wavelet transform based principal component averaging fusion for medical images. AEU - International Journal of Electronics and Communications. 2015; 69(6): 896-902.

9. Liu Y, Yu F. An automatic image fusion algorithm for unregistered multiply multi-focus images. Optics Communications. 2014; 341:101-13.

10. Singh G, Singh G, Aujla GS. MHWT-A Modified Haar Wavelet Transformation for Image fusion. International Journal of Computer Applications. 2013; 79(1): 26-31.

11. Clonda D, Lina J, Goulard B. Complex Daubechies wavelets: properties and statistical image modelling. Signal Processing. 2004; 84(1): 1-23.

12. Zitova B, Flusser J. Image registration methods: A survey. Image and Vision Computing. 2003; 21(11): 977-1000.

13. Huang W, Jing Z. Evaluation of focus measures in multifocus image fusion. Pattern Recognition Letters. 2007; 28(4): 493-500.

14. Lowe D. Distictive image features from Scale-Invariant keypoints. International Journal of Computer Vision. 2004; 60(2): 91-110.

15. Ce Liu, Yuen J, Torralba A. SIFT Floe: Dense Correspondence across scenes and its applications. IEEE Transactions on Pattern Analysis and Machine Intelligence. 2011; 33(5):978-94.

16. Fischler M, Bolles R. Random sample consensus: a paradigm for model fitting with applications to image analysis and automated cartography. Communications of the ACM. 1981; 24(6):381-95. 